\title{
RESERVE PRICE OF RISK-AVERSE SEARCH ENGINE IN KEYWORD AUCTIONS WITH ADVERTISERS' ENDOGENOUS INVESTMENT
}

\author{
Qin Yang ${ }^{1}$, Xianpei Hong ${ }^{2}$, Zongunn WAnG ${ }^{3, *}$ And Huaige Zhang ${ }^{4}$
}

\begin{abstract}
Motivated by vigorous development of keyword auctions, this paper analyzes the reserve price policies in keyword auction with advertisers' endogenous investment and risk-averse search engine. We explore advertisers' optimal investment and equilibrium bidding strategies and derive the determination functions where utility-maximizing reserve price and efficient reserve price which maximizes the social welfare satisfy respectively. The results show that advertisers' equilibrium bidding is monotonously increasing in bidders' valuations, the number of advertisers, as well as the reserve price. Meanwhile, advertisers' optimal investment is negatively correlated with reserve price and the number of advertisers. By numerical examples, the utility-maximizing reserve price decreases with the risk aversion parameter and the number of advertisers. Search engine's expected utility increases with risk aversion parameter and decreases with the number of advertisers. Moreover, we declare that search engine can use reserve price as a regulatory tool to increase the utility. But there is an upper bound on search engine's utility. It is interesting to find the efficient reserve price equals to zero. Hence there is a trade-off between total efficiency and search engine's utility by search engine that has incentive to withhold reserve price that would benefit social welfare.
\end{abstract}

Received June 26, 2018. Accepted November 7, 2019.

\section{INTRODUCTION}

As a commodity trading mechanism, auctions refer to the behavior of a market organization that uses a set of explicit mechanisms to determine resource allocation and price on the basis of the bid of its participants, which is widely used in economic activity [23]. Since Friedman [15] studied on auction theory and Vickrey [30] put forward a benchmark auction model and Revenue Equivalence Theorem, auction theory formed and obtained a wealth of research.

With the flourishing development of Internet, search engines are indispensable tools for Internet users to search effective information. Search engines use keyword auctions to promote commercial advertisements and obtain high profits, which is an innovative application of the traditional auction on sponsored search. The sponsored search market maintains rapid growth with its highly-directed and non-invasive forms of presentation [1], which has brought enormous business opportunities and huge revenues for search engines [17]. For example, search

Keywords. Keyword auctions, reserve price, risk aversion, endogenous investment, social welfare.

1 School of Economics and Management, Hubei University of Technology, Wuhan 430068, P.R. China.

2 College of Economics and Management, Huazhong Agricultural University, Wuhan 430070, P.R. China.

3 School of Management, Huazhong University of Science and Technology, Wuhan 430074, P.R. China.

4 School of Business Administration, Guangdong University of Finance \& Economics, Guangzhou 510320, P.R. China.

*Corresponding author: wangz_hust@163.com 
engine giants Google in US and Baidu in China, their growing revenues are almost entirely due to advertising. According to Google's financial report, Google's advertising revenue in 2016 was $\$ 79.383$ billion, accounting for $88.5 \%$ of the total revenue. China's search engine Baidu's largest revenue source was online advertising, reaching $¥ 64.525$ billion and accounting for $91.46 \%$ of total revenue.

The profitability of keyword auction and high penetration rate of the advertising model led advertisers to disregard the improvement of their performance, even provide low-relevance information and false advertising. In 2011, Google was identified as advertising an illegal Canadian online pharmacy and paid a $\$ 500$ million fine. This situation will not only affect the search experience of users, but also adversely disrupt the benign competition of advertisers. In the long run, it will certainly lead to the loss of reputation and revenue of search engines, which is not conducive to the sustainable development of the sponsored search market.

As search engine's business model experiences the continuous innovation, keyword auction mechanisms are constantly promoting. With search engines' increasingly attention on advertisers' performance [4] and strong government supervision, advertisers will engage in extensive market research or experiments to optimize web page design and improve service quality, aiming at a better rank in search engine results page and ultimately having higher clicks [27]. Researches on investing for upgrading "quality" are adequate in the field of supply chain [18] and government reverse auctions [3,5]. For example, Branco [5] researched the government procurement auction in which two companies bid for government projects, and inefficient bidders invested to improve its technical level in order to tender for the project successfully (increase R\&D expenditure, capital investment, etc.). Schlosser et al. [27] investigated the impact of web investments on search consumers' beliefs and online purchase intentions and declared that website investment (including time, financial resources, and various efforts) was strongly related to the user's willingness to buy. Our present paper is much related in spirit to Chen et al. [7]. They took the lead in merging investment into keyword auctions where bidders with a low performance level can improve their performance at a certain cost. The investment in their study is a fixed parameter which is exogenously determined for simplification, however, advertisers' investment is continuously changing in reality. In order to emphasize the role played by investment, we consider a more practical setting in which advertisers' investment is endogenously determined. Investment in our study specifically refers to investing in strengthening website design, optimizing web interface and providing plentiful and relevant content. This will certainly promote advertisers' performance which is represented by the total clicks gained from search users. Obviously, the continuously changing investment will inevitably pay financial costs. In this way, the optimal decision on investment depends on the tradeoff between the increase in revenue (because of the promotion of clicks) and the investment cost. As sponsored adverting market matures gradually, search engines adopting a ranking mechanism which takes advertisers' endogenous investment into consideration will promote the virtuous circle of the keyword advertising industry. Exploring advertisers' optimal investment decisions under endogenous investment extends the previous research, which is one innovation in this article.

In economic analysis, uncertainty is associated with risk. In auctions, bidders and sellers usually encounter uncertainty $[8,32]$. Pratt [25] formally proposed the concept of risk aversion to explain the agent's behavior in uncertain situations. In practice, participants are reluctant to confront uncertainty and have the characteristics of risk aversion when the subject matter is highly valued [13]. So, the sellers in auction (search engine in keyword auction) might be risk-averse. There are certain theoretical foundations for the study of auction model (e.g. [10]) and optimal mechanism design under risk aversion. Early researches introduced risk-averse bidders' strategies $[21,26]$ or compared the bidder's preference for the auction model from the perspective of risk-averse bidders [22]. $\mathrm{Lu}$ and Perrigne [20] used real-world data from Timber Auctions to assess the risk aversion of bidders. Fang and Tang [14] demonstrated that bidders' risk attitudes have key implications for the choices of revenue-maximizing auction formats. Accordingly, researches on risk-averse sellers have gradually emerged. Waehrer and Rothkopf [31] discussed risk-averse seller's preferences for auction models and analyzed the trend of seller's optimal reserve price on seller's risk aversion parameter. Hu et al. [16] extended Riley and Samuelson's study [26] to explore how risk aversion parameter affects the optimal reserve price under the assumption that both bidders and auctioneers are risk averse. There are quite adequate researches on risk aversion in traditional auction theory, while extending the risk neutrality to risk aversion in keyword auction needs prompt development. Inspired by 
these previous researches, we consider that the risk-neutral assumption of search engine in keyword auction is not necessarily satisfied, then risk-averse search engine is introduced. The introduction of risk aversion attitude is more practical and can better reflect the diversity of search engine preferences, which is another innovation of our research.

Reserve price is one of the important parameters involved in traditional auction theory, which is the main tool for sellers to maximize the expected revenue. Riley and Samuelson [26] discussed the necessary condition for the seller's optimal reserve price in the first-price sealed auction. McAfee and McMillan [23] showed that the seller's reasonable setting of reserve price can exclude bidders whose bid is lower than reserve price, which is beneficial to the increase of seller's profit. Stephanie and Patrick [28] found that the number of bidders participating in the auction would affect the optimal reserve price. Hu et al. [16] assumed both buyers and sellers are risk averse, and studied the influence of risk aversion on optimal reserve price setting. In keyword auction, how search engine sets the optimal reserve price is a theoretical hotspot and conundrum. Chen et al. [6] gave an optimal reserve price that satisfies the maximization of search engine revenue. Edelman and Schwarz [12] proposed the conditions for satisfying the optimal reserve price in generalized English auctions. Ostrovsky and Schwarz [24] made use of the data of Yahoo's sponsored search auction to study reserve price with empirical methods and demonstrated that setting an appropriate reserve price can increase Yahoo's revenue, but a high reserve price may prevent advertisers from participating in online advertising and make auction invalid. Liu et al. [19] designed a differentiated reserve price strategy for asymmetric advertisers (dividing advertisers into high and low types) which can improve search engine's revenue and auction efficiency. In fact, reserve price of auctioned slots not only has an important impact on the auctioneer's revenue, but also affects the bidder's payoff and auction efficiency. Therefore, a reasonable reserve price is beneficial for allocation of slots and conducive to the healthy development of the keyword auctions market. The third innovation of our study on reserve price is that we explore how advertisers' investment behavior and search engine's risk aversion influence the reserve price setting, which expands the theoretical research on the field of reserve price in keyword auctions in order to provide scientific decision-making for search engine's reserve price policies.

The study on keyword auction also focuses on auction efficiency which maximizes the social welfare. Aggarwal et al. [2] pointed out that, from search engine's perspective, the most basic question is how to maximize the search engine's own revenue and the auction efficiency. Chen et al. [7] discussed search engine's auction rules which maximize social welfare. Liu et al. [19] explored the efficient reserve price and revenue-maximizing reserve price. Kelly et al. [17] provided an efficient mechanism that maximizes social welfare in pay-per-click market. Sundararajan and Talgam-Cohen [29] demonstrated efficiency-optimal and revenue-optimal mechanisms in adverting auctions. For search engines, there is a trade-off between current earnings (revenue-maximizing policy) and long-term development (efficient policy). Our paper takes efficient reserve price into consideration to guide search engine to focus on long-term interests, establish a balance between commercial interests and social welfare, and promote the sustainable development of the sponsored search market.

The remainder of the paper is organized as follows. We describe our model in Section 2. In Section 3, we investigate advertiser's investment decisions and equilibrium bidding strategies. The utility-maximizing reserve price and efficient reserve price of risk-averse search engine are presented in Section 4. Numerical examples are discussed in Section 5. Section 6 concludes the paper and discusses further research directions.

\section{Model Setup}

Assumption and parameter setting are as follows.

(1) According to Chen et al. [7], we assume that search engine sells only one available advertising slot to $n$ advertisers. This situation that the search engine result page has only one advertising slot is common in reality.

(2) Advertisers' valuation of slot measures the value that a single click can bring to advertisers. Slot valuation is private information which is unknown to the search engine and other advertisers. The unit valuation $v$ is independent and identically distributed on the interval $[0,1]$, following a density function $f(v)$ which is 
positive and continuous all over the interval. Let $F(v)$ denote the corresponding probability distribution function, which is common knowledge. Assume that all advertisers are risk-neutral.

(3) We denote $e(e \in[0, \infty))$ as advertisers' investment. In practice, due to the different management and experience, the ability of using the same resources is inconsistent. For simplification, this paper sets that advertisers pay the same cost for the same investment, and then the cost function can be uniformly written as $c(e)$. It's clear that $c(e) \geq 0$ and it increases in $e$. We set $c(e)$ as a convex function as prior literatures commonly do.

(4) We portrayed endogenous investment into clicks where advertisers' investment can enhance the total hits. It's based on the following considerations.

Firstly, from a practical perspective, it is reasonable that paying investment will enhance the quality of web and website content of the advertisers, which can attract more users to click and consolidate original hits. Then total clicks increases inevitably.

Secondly, we know the cost function is increasing in investment. The article discusses the continuous variable $e$ which must be portrayed into our model, so preferential treatment on the number of clicks should be given for their investment.

Thirdly, competitive investment which portrayed in total clicks will impel advertisers putting more investment. This is consistent with the reality.

Obviously, the total clicks $y(e)$ is an increasing function of $e$ and a concave function.

(5) Assume that search engine is risk averse. Set the search engine's utility function is $u(\cdot)$, and satisfies $u^{\prime}(\cdot)>0$, $u^{\prime \prime}(\cdot)<0$ and $u(0)=0$. The introduction of risk aversion complicates the auction problem and brings great difficulties to the analysis. Therefore, most literatures appoint a specific utility function to reflect the risk attitude of participants. For example, Cox et al. [9] introduced CRRA (constant relative risk aversion) utility function which contains a risk-neutral situation, and can reasonably explain the participants' risk aversion [20]. It is widely used in a series of research on risk aversion's representation. Imitating this approach, we assume search engine's utility function is CRRA, and can be expressed as $u(x)=x^{1-r_{s}} \cdot r_{s} \in[0,1)$ is the relative degree of risk aversion of search engine. The higher the $r_{s}$, the higher the degree of risk aversion. When $r_{s}=0$, it means search engine is risk-neutral.

(6) Let the search engine's reserve price be $R$, the advertisers whose valuation $v$ is lower than the reserve price will not participate in the keyword auction.

This model can be defined as follows.

We consider first-price, sealed-bid unit-price auction where the search engine is risk-averse in a symmetric independent private value model. Firstly, search engine announces the reserve price. Next, in the investment stage, the endogenous investment is introduced to analyze the optimal investment strategy of advertisers. Then, in bidding stage, the equilibrium bidding strategies of advertisers are explored. Subsequently, based on the advertiser's investment and bidding behavior, the search engine can choose the best reserve price based on either maximizing the expected utility of search engine or maximizing social welfare, and selects the winning advertiser based on the auction rules.

\section{AdVERTiseR's INVESTMENT AND BIDDING DECISION}

In this section, given the reserve price of search engine, we use backward induction to address the issue of advertisers' investment and bidding behavior. First of all, we derive advertisers' equilibrium bidding function in bidding stage, and then plug the optimal bidding function into investment stage to explore advertisers' investment behavior. Now, we begin with the analysis of advertisers' bidding strategies.

\subsection{Advertisers' equilibrium bidding strategies}

We conjecture that the equilibrium bidding function is monotonously increasing in the advertisers' valuation (This will be verified later.). Since advertiser's bidding function is strictly increasing in $v$, advertiser wins this slot only when all other advertisers' valuation is less than $v$. Then, the probability of winning for advertisers can 
be represented by $\rho(v)=[F(v)]^{n-1} \cdot y(e)$ expresses the amount of click after putting investment $e$ in bidding stage.

Let $V(v)$ be the expected equilibrium payoff of advertisers with valuation $v$ under bidding stage, then $V(v) \equiv$ $U(y, v, b(v))=y(e) \cdot(v-b(v))[F(v)]^{n-1}$.

We consider the symmetric perfect Bayesian pure strategy Nash equilibrium, namely: advertisers whose valuation is identical will bid the same in equilibrium [7]. According to the conclusion of the traditional auction theory, a symmetric Bayesian Nash equilibrium strategy exists in the first-price auction, i.e. $v \in[0,1], b_{i}(v)=$ $b_{j}(v)=b(v)$. Then, the Bayesian Nash equilibrium condition is equivalent to the following condition: if the private value of an advertiser is $v$, it exists $x \in[0,1]$ which maximizes the advertiser's equilibrium expectation of payment $V(v, x)$. The risk-neutral advertiser's equilibrium expected payment is specifically expressed as:

$$
V(v, x) \equiv U(y, v, b(x))=y(e) \cdot(v-b(x))[F(x)]^{n-1} .
$$

The equilibrium of advertisers' bidding strategy is characterized in Proposition 3.1.

Proposition 3.1. Given the reserve price $R$, the equilibrium bidding function of advertisers is $b(v)=v-$ $\frac{\int_{R}^{v}[F(t)]^{n-1} \mathrm{~d} t}{[F(v)]^{n-1}}$, and $b(v)$ is monotonously increasing in $v$.

Proof. According to the first-order conditions of the optimal solution, we have $\left.\frac{\mathrm{d} u(x, v)}{\mathrm{d} x}\right|_{x=v}=0$. Combining with $(3.1), b^{\prime}(v)=(n-1) \cdot \frac{f(v)}{F(v)} \cdot(v-b(v))$ can be obtained.

Advertiser's equilibrium bidding strategy satisfies first-order linear ordinary differential equation. Since the keyword auction contains the reserve price $R, b(R)=R$ is the initial condition. We can derive

$$
b(v)=v-\frac{\int_{R}^{v}[F(t)]^{n-1} \mathrm{~d} t}{[F(v)]^{n-1}}, \quad \text { for } v \in[R, 1] .
$$

When the advertiser's valuation is less than the reserve price $R$, the bid is zero.

Based on the above analysis, the advertiser's equilibrium bidding function can be expressed as:

$$
b(v)=\left\{\begin{array}{ll}
v-\frac{\int_{R}^{v}[F(t)]^{n-1} \mathrm{~d} t}{[F(v)]^{n-1}}, & v \in[R, 1] \\
0, & v \in[0, R)
\end{array} .\right.
$$

Since $\frac{\mathrm{d} b(v)}{\mathrm{d} v}=(n-1) \cdot[F(v)]^{-n} \cdot f(v) \cdot \int_{R}^{v}[F(t)]^{n-1} \mathrm{~d} t>0$, for $v \in[R, 1], b(v)$ is monotonously increasing in $v$.

Proposition 3.1 reveals that the advertiser's equilibrium bid is positively related to the valuation, and the bid is always less than the valuation. It's worth noting that advertisers' investment and bidding decisions are independent of each other, because the investment $e$ can be identified as sunk cost and do not affect advertisers' current bidding decisions.

In accordance with $\frac{\mathrm{d} b(v)}{\mathrm{d} v}=(n-1) \cdot[F(v)]^{-n} \cdot f(v) \cdot \int_{R}^{v}[F(t)]^{n-1} \mathrm{~d} t, \frac{\partial\left(\frac{\mathrm{d} b(v)}{\mathrm{d} v}\right)}{\partial R}=-(n-1) \cdot[F(v)]^{-1} \cdot f(v)$. $\left[\frac{F(R)}{F(v)}\right]^{n-1}<0$ can be derived.

It is interesting to find that the more the reserve price is, the more slowly the bid function increases in bidder's value. This indicates that the rate at which the advertiser's bidding increases in a bidder's value decreases in search engine's reserve price, which is consistent with the conclusion of $\mathrm{Hu}$ et al. [16] in traditional auction research.

On the basis of the equilibrium bidding function (3.2), it is easy to declare that the number of advertisers and the reserve price of search engine have an impact on the equilibrium bidding, which is described specifically in Corollary 3.2. 
Corollary 3.2. Advertiser's bidding function $b(v)$ increases in both the number of advertisers and search engine's reserve price.

Proof. Taking the first-order derivative of (3.2) with respect to $n$, we have

$$
\begin{aligned}
& \frac{\partial b(v, n)}{\partial n}=-\int_{R}^{v}\left[\frac{F(t)}{F(v)}\right]^{n-1} \cdot \ln \frac{F(t)}{F(v)} \mathrm{d} t \\
& \forall t \in[0, v], \quad \frac{F(t)}{F(v)}<1, \quad \text { then } \ln \frac{F(t)}{F(v)}<0, \quad \text { so } \frac{\partial b(v, n)}{\partial n}>0 .
\end{aligned}
$$

Taking the first-order derivative of (3.2) with respect to $R$, we get

$$
\frac{\partial b(v)}{\partial R}=(F(v))^{-(n-1)} \cdot(F(R))^{n-1}>0 .
$$

Therefore, with the increase of the number of advertisers or search engine's reserve price, advertisers bid more aggressively. The number of advertisers can be considered as the market size of the bidders, which can reflect the degree of competition among advertisers to some extent. The increase in the number of advertisers will reduce the probability of advertisers' winning the slot, which induce advertisers to increase the bid for improving the winning probability. In this way, the fiercer competition will inevitably increase the advertiser's bid. Similarly, the higher the reserve price set by the search engine, the higher the bid of the advertiser is obviously.

In consideration of $\frac{\partial b(v)}{\partial R}=(F(v))^{-(n-1)} \cdot(F(R))^{(n-1)}$, the rate at which advertisers' bid increases in reserve price is related to the number of advertisers. Taking the first-order derivative of $\frac{\partial b(v)}{\partial R}$ with respect to $n$, we have

$$
\frac{\partial\left(\frac{\partial b(v)}{\partial R}\right)}{\partial n}=\frac{\partial\left(\left(\frac{F(R)}{F(v)}\right)^{n-1}\right)}{\partial n}=\left(\frac{F(R)}{F(v)}\right)^{n-1} \cdot \ln \left(\frac{F(R)}{F(v)}\right)<0 .
$$

Consequently, the rate at which the advertiser's bid increases in the reserve price decreases with the number of advertisers. That is, the greater the number of advertisers, the lower the advertiser's bid increment caused by the increase in reserve price. This is crucial for the later analysis.

In summary, regardless of the distribution function the private value follows, the advertisers' equilibrium bid increases in their valuation, the number of advertisers and the search engine's reserve price. It is worth noting that the degree of search engine's risk aversion is not shown in the advertiser's bidding function, that is, the risk aversion of search engine does not directly affect the advertiser's bidding strategy, but it will indirectly influence the advertiser's bidding strategy by affecting the reserve price of the search engine.

\subsection{The optimal investment decisions}

In investment stage, we focus on the investment decisions of advertisers where search engine's reserve price and advertisers' equilibrium bidding strategies in bidding stage are given.

The investment $e$ is a continuous variable in our study, and advertisers choose the optimal maximum investment based on maximizing expected payoff in investment stage. The optimal investment $e^{*}$ of advertisers can be educed in the following Proposition 3.3.

Proposition 3.3. Advertisers' optimal investment $e^{*}$ which maximizes the expected payoff is determined by the following equation: $y^{\prime}\left(e^{*}\right) \cdot \int_{R}^{v}[F(t)]^{n-1} \mathrm{~d} t-c^{\prime}\left(e^{*}\right)=0$, and $e^{*}(v)$ monotonically increases with the valuation $v$. 
Proof. The advertiser's maximization problem of expected payoff is described as $\max _{e}\{y(e) \cdot(v-b(v)) \cdot \rho(v)-c(e)\}$, $e \in[0, \infty)$.

Substituting $b(v), \rho(v)$ into the expression of the optimization problem above, we get

$$
\max _{e}\left\{y(e) \cdot \int_{R}^{v}[F(t)]^{n-1} \mathrm{~d} t-c(e)\right\}, \quad e \in[0, \infty) .
$$

Since $c(e)$ is convex and $y(e)$ is concave, then we have $c^{\prime \prime}(e)>0$ and $y^{\prime \prime}(e)<0$, therefore $y^{\prime \prime}(e)$. $\int_{R}^{v}[F(t)]^{n-1} \mathrm{~d} t-c^{\prime \prime}(e) \leq 0$. The objective function is a concave function, and this optimization problem inevitably has a unique optimal solution. So, advertisers' optimal investment exists and is unique. Taking the first-order condition of the expected payoff with respected to $e$, we obtain the optimal solution $e^{*}$ satisfies the equation below:

$$
y^{\prime}\left(e^{*}\right) \cdot \int_{R}^{v}[F(t)]^{n-1} \mathrm{~d} t-c^{\prime}\left(e^{*}\right)=0 .
$$

Obviously, advertisers' optimal investment is a function with valuation. Substituting $e^{*}(v)$ into (3.3) and taking the first-order derivative with respect to $v$, we have

$$
y^{\prime \prime}\left(e^{*}\right) \cdot \frac{\partial e^{*}}{\partial v} \cdot \int_{R}^{v}[F(t)]^{n-1} \mathrm{~d} t+y^{\prime}\left(e^{*}\right) \cdot[F(v)]^{n-1}-c^{\prime \prime}\left(e^{*}\right) \cdot \frac{\partial e^{*}}{\partial v}=0 .
$$

It can be reorganized as:

$$
\left[c^{\prime \prime}\left(e^{*}\right)-y^{\prime \prime}\left(e^{*}\right) \cdot \int_{R}^{v}[F(t)]^{n-1} \mathrm{~d} t\right] \cdot \frac{\partial e^{*}}{\partial v}=y^{\prime}\left(e^{*}\right) \cdot[F(R)]^{n-1} .
$$

The right-hand side of the equation above is positive and the coefficient of $\frac{\partial e^{*}}{\partial v}$ is positive (due to $c^{\prime \prime}(e)>0$ and $\left.y^{\prime \prime}(e)<0\right)$, then $\frac{\partial e^{*}}{\partial v}>0$ holds.

Proposition 3.3 indicates that advertisers with higher valuations tend to disburse greater investment. Keeping other conditions constant, the marginal return from investing with higher valuation will be greater than the marginal cost, so that advertisers are willing to make additional investments to maximize their expected payoff. Among them, when the advertiser's valuation is just equal to reserve price $R$, we have $e^{*}(v=R)=0$.

Obviously, from (3.3) we can infer that search engine's reserve price and the number of advertisers can exert an influence on the optimal investment, which can be summarized in Corollary 3.4.

Corollary 3.4. The effects of search engine's reserve price and the number of advertisers on the optimal investment are as follows:

(i) The advertiser's optimal investment $e^{*}$ is monotonically decreasing in the reserve price.

(ii) The advertiser's optimal investment $e^{*}$ is monotonically decreasing in the number of advertisers.

Proof. Substituting $e^{*}(R)$ into (3.3), and taking the first-order derivative in (3.3) with respect to $R$, we have

$$
y^{\prime \prime}\left(e^{*}\right) \cdot \frac{\partial e^{*}}{\partial R} \cdot \int_{R}^{v}[F(t)]^{n-1} \mathrm{~d} t+y^{\prime}\left(e^{*}\right) \cdot\left[-[F(R)]^{n-1}\right]-c^{\prime \prime}\left(e^{*}\right) \cdot \frac{\partial e^{*}}{\partial R}=0 .
$$

After reorganization, we have

$$
\left\{c^{\prime \prime}\left(e^{*}\right)-y^{\prime \prime}\left(e^{*}\right) \cdot \int_{R}^{v}[F(t)]^{n-1} \mathrm{~d} t\right\} \cdot \frac{\partial e^{*}}{\partial R}=y^{\prime}\left(e^{*}\right) \cdot\left[-[F(R)]^{n-1}\right] .
$$

The right-hand side of the equation above is negative and the coefficient of $\frac{\partial e^{*}}{\partial R}$ is positive (since $c^{\prime \prime}(e)>0$ and $\left.y^{\prime \prime}(e)<0\right)$, then $\frac{\partial e^{*}}{\partial R}<0$ holds. 
Meanwhile, we substitute $e^{*}(n)$ into (3.3), and take the first-order derivative with respect to $n$ :

$$
y^{\prime \prime}\left(e^{*}\right) \cdot \frac{\partial e^{*}}{\partial n} \cdot \int_{R}^{v}[F(t)]^{n-1} \mathrm{~d} t+y^{\prime}\left(e^{*}\right) \cdot \int_{R}^{v}[F(t)]^{n-1} \cdot \ln [F(t)] \mathrm{d} t-c^{\prime \prime}\left(e^{*}\right) \cdot \frac{\partial e^{*}}{\partial n}=0 .
$$

Then, we get

$$
\left\{c^{\prime \prime}\left(e^{*}\right)-y^{\prime \prime}\left(e^{*}\right) \cdot \int_{R}^{v}[F(t)]^{n-1} \mathrm{~d} t^{1-r}\right\} \cdot \frac{\partial e^{*}}{\partial n}=y^{\prime}\left(e^{*}\right) \cdot \int_{R}^{v}[F(t)]^{n-1} \cdot \ln [F(t)] \mathrm{d} t .
$$

Since $\ln [F(t)]<0$, the right-hand side of the equation above is less than zero and the coefficient of $\frac{\partial e^{*}}{\partial n}$ is greater than zero (due to $c^{\prime \prime}(e)>0$ and $y^{\prime \prime}(e)<0$ ), then $\frac{\partial e^{*}}{\partial n}<0$ holds.

Corollary 3.4 shows that the higher the search engine's reserve price, the higher the threshold required for advertisers. Then, the marginal revenue generated by the investment will be reduced, which is less than the marginal cost, so that advertisers will reduce investment and make the marginal return equal to the marginal cost for maximizing their own expected payoff. With other conditions unchanged, the greater the number of advertisers, the fiercer that competition will be, and the marginal cost of increasing investment will be greater than the marginal revenue obtained. At this time, advertisers will reduce investment to maximize their own payoff.

It is worth noting that the results in Proposition 3.3 and Corollary 3.4 are independent of which distribution the advertiser's valuation follows. Similarly, search engine's risk aversion is not shown in the expression of advertiser's optimal investment, that is, the search engine's risk aversion has no direct influence on the advertiser's optimal investment decision, but indirectly influences advertiser's investment strategy by affecting the search engine's reserve price.

\section{RISK-AVERSE SEARCH ENGINE'S RESERVE PRICE}

In this section, we examine the reserve price policies of risk-averse search engine under utility-maximizing consideration and efficient consideration (maximizing the social welfare) respectively.

\subsection{The utility-maximizing reserve price}

In the field of risk decision making, decision makers often pursue maximum expected utility, instead of expected revenue. From the assumption that the utility function of the risk-averse search engine is $u(x)=x^{1-r_{s}}$, under reserve price $R$, the expected utility can be expressed as

$$
U_{\mathrm{SE}}=\sum_{i} y_{i}(e) \cdot \rho_{i}(v) \cdot\left[b_{i}(v)\right]^{1-r_{s}}=\int_{R}^{1} y(e) \cdot F^{n-1}(v) \cdot\left[v-\frac{\int_{R}^{v}[F(t)]^{n-1} \mathrm{~d} t}{[F(v)]^{n-1}}\right]^{1-r_{s}} \cdot f(v) \mathrm{d} v .
$$

Considering the advertiser's endogenous investment and risk-averse search engine brings great complexity of the model. For simplicity, let $F(v)=v$ (i.e. advertisers' value follows uniform distribution at $[0,1]$ ). In this case, $b(v)=\frac{n-1}{n} v+\frac{1}{n} v^{1-n} \cdot R^{n}$, and $U_{\mathrm{SE}}$ is simplified as

$$
U_{\mathrm{SE}}=\int_{R}^{1} y(e) \cdot v^{n-1} \cdot\left(\frac{n-1}{n} v+\frac{R^{n}}{n} v^{1-n}\right)^{1-r_{s}} \mathrm{~d} v .
$$

Then, we theoretically derive the optimal reserve price that maximizes search engine's utility. Taking the first-order derivative of $U_{\mathrm{SE}}$ in (4.1) with respect to $R$, we have

$$
\begin{aligned}
\frac{\mathrm{d} U_{\mathrm{SE}}}{\mathrm{d} R}= & -y[e(v=R)] \cdot R^{n-r_{s}}+\int_{R}^{1} \frac{\partial y(e)}{\partial e} \cdot \frac{\partial e}{\partial R} \cdot v^{n-1} \cdot\left(\frac{n-1}{n} v+\frac{R^{n}}{n} v^{1-n}\right)^{1-r_{s}} \mathrm{~d} v \\
& +\int_{R}^{1} y(e) \cdot\left(1-r_{s}\right) \cdot\left(\frac{n-1}{n} v+\frac{R^{n}}{n} v^{1-n}\right)^{-r_{s}} \cdot R^{n-1} \mathrm{~d} v .
\end{aligned}
$$


where $e^{*}$ satisfies the equation (3.3). Substitute $F(v)=v$ into (3.3), we get

$$
y^{\prime}\left(e^{*}\right) \cdot \frac{v^{n}-R^{n}}{n}-c^{\prime}\left(e^{*}\right)=0
$$

We can derive $\frac{\partial e^{*}}{\partial R}=-\frac{y^{\prime}\left(e^{*}\right) \cdot R^{n-1}}{c^{\prime \prime}\left(e^{*}\right)-y^{\prime \prime}\left(e^{*}\right) \cdot \frac{v^{n}-R^{n}}{n}}$ from (4.3), which will be used in (4.2).

Incorporating (4.2) and (4.3), we have the optimal utility-maximizing reserve price $R_{\mathrm{SE}}^{*}$ in Proposition 4.1.

Proposition 4.1. The utility-maximizing reserve price $R_{\mathrm{SE}}^{*}$ of the risk-averse search engine is determined by following equations combined with (4.2) and (4.3).

The proof and related analysis are as above.

Evidently, search engine's utility-maximizing reserve price is related to the number of advertisers and the degree of search engine's risk aversion. After the introduction of advertisers' endogenous investment, the optimal investment and the utility-maximizing reserve price of search engine will influence each other, which increases the complexity of analyzing their relationship. Therefore, a comparative static analysis of the utility-maximizing reserve price on the number of advertisers and the degree of search engine's risk aversion will be extended in the numerical analysis.

As mentioned above, under the reserve price $R$, the expected utility of risk-aversion search engine is expressed in (4.1).

It becomes unclear how the number of advertisers and search engine's risk aversion affect the search engine's utility. It is compositely determined by the form of the click function and cost function, the number of advertisers, the degree of search engine's risk aversion, as well as the reserve price. Further analysis will be performed in numerical simulations.

\subsection{The efficient reserve price}

Social welfare refers to the total expected payoff of auctioneers and bidders which can reflect the auction efficiency to some extent. Auctioneer intends to maximize social welfare in mechanism design is the best choice in the long run for substantial development of sponsored search market.

Under the reserve price $R$, the social welfare can be expressed as:

$$
\begin{aligned}
U_{\mathrm{SW}}= & \sum_{i} y_{i}(e) \cdot \rho_{i}(v) \cdot\left[b_{i}(v)\right]^{1-r_{s}}+\sum_{i} y_{i}(e) \cdot \rho_{i}(v) \cdot\left(v-b_{i}(v)\right)-\sum_{i} c_{i}(e) \\
= & \int_{R}^{1} y(e) \cdot F^{n-1}(v) \cdot\left[v-\frac{\int_{R}^{v}[F(t)]^{n-1} \mathrm{~d} t}{[F(v)]^{n-1}}\right]^{1-r_{s}} \cdot f(v) \mathrm{d} v \\
& +\int_{R}^{1} y(e) \cdot F^{n-1}(v) \cdot \frac{\int_{R}^{v}[F(t)]^{n-1} \mathrm{~d} t}{[F(v)]^{n-1}} \cdot f(v) \mathrm{d} v-\int_{R}^{1} c(e) \cdot f(v) \mathrm{d} v .
\end{aligned}
$$

Let $F(v)=v$ (i.e. advertisers' value follows uniform distribution at $[0,1]), U_{\mathrm{SW}}$ is simplified as

$$
U_{\mathrm{SW}}=\int_{R}^{1} y(e) \cdot v^{n-1} \cdot\left(\frac{n-1}{n} v+\frac{R^{n}}{n} v^{1-n}\right)^{1-r_{s}} \mathrm{~d} v+\int_{R}^{1} y(e) \cdot v^{n-1} \cdot\left(\frac{1}{n} v-\frac{R^{n}}{n} v^{1-n}\right) \mathrm{d} v-\int_{R}^{1} c(e) \mathrm{d} v .
$$

Then, we theoretically derive the efficient reserve price that maximizes social welfare. Taking the first-order derivative of $U_{\mathrm{SW}}$ in (4.4) with respect to $R$, we have

$$
\begin{aligned}
\frac{\mathrm{d} U_{\mathrm{SW}}}{\mathrm{d} R}= & -y(e(R)) \cdot R^{n-r_{s}}+c(e(R)) \\
& +\int_{R}^{1}\left[\frac{\partial y(e)}{\partial e} \cdot v^{n-1} \cdot\left(\frac{n-1}{n} v+\frac{R^{n}}{n} v^{1-n}\right)^{1-r_{s}}+\frac{\partial y(e)}{\partial e} \cdot v^{n-1} \cdot\left(\frac{1}{n} v-\frac{R^{n}}{n} v^{1-n}\right)-\frac{\partial c(e)}{\partial e}\right] \cdot \frac{\partial e}{\partial R} \mathrm{~d} v
\end{aligned}
$$




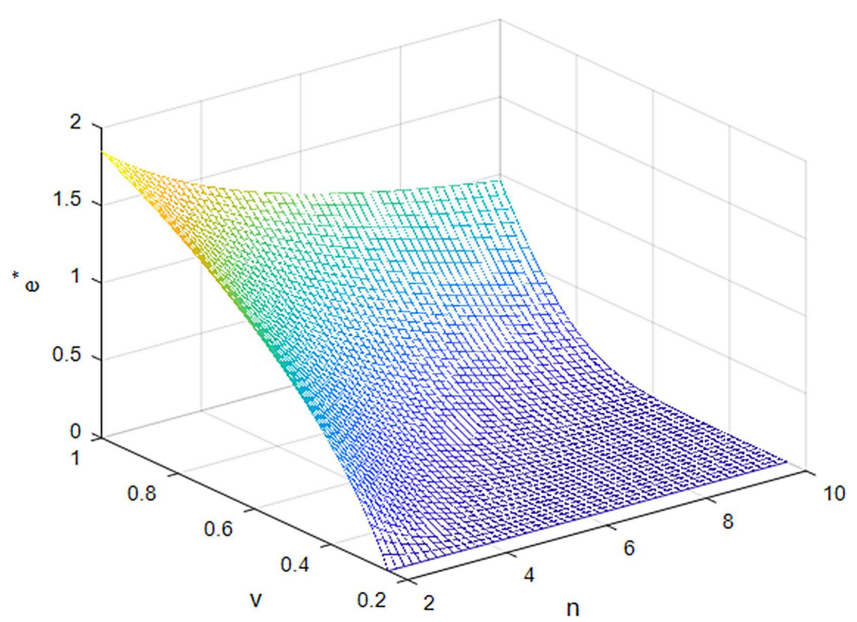

Figure 1. The optimal investment with $v$ and $n(R=0.25)$.

where $e^{*}$ meets $y^{\prime}\left(e^{*}\right) \cdot \frac{v^{n}-R^{n}}{n}-c^{\prime}\left(e^{*}\right)=0$ when $F(v)=v$.

So, we also have $\frac{\partial e^{*}}{\partial R}=-\frac{y^{\prime}\left(e^{*}\right) \cdot R^{n-1}}{c^{\prime \prime}\left(e^{*}\right)-y^{\prime \prime}\left(e^{*}\right) \cdot \frac{v^{n}-R^{n}}{n}}$.

Incorporating (4.3) and (4.5), we have the efficient reserve price for maximizing social welfare $R_{\mathrm{SW}}^{*}$ in Proposition 4.2 .

Proposition 4.2. The efficient reserve price $R_{\mathrm{SW}}^{*}$ which maximizes social welfare is determined by following equations combined with (4.3) and (4.5).

The proof and related analysis are as above. Similarly, the analysis of $R_{\mathrm{SW}}^{*}$ is relatively complex and will be explained in the numerical examples.

\section{NUMERICAL ANALYSIS}

In this section, we study the optimal investment and different reserve price policies, as well as the search engine's utility and social welfare by numerical examples. Let advertisers' value follows uniform distribution at $[0,1]$, i.e. $F(v)=v$. The advertiser's equilibrium bidding function is $b(v)=\frac{n-1}{n} v+\frac{1}{n} v^{1-n} \cdot R^{n}$. Assume the cost function and the click function with the investment are $c(e)=\frac{e^{2}}{50}$ and $y(e)=2-\exp (-e)$, respectively.

\subsection{Analysis of the advertisers' optimal investment}

Substituting the parameter settings above into (3.3), the advertiser's optimal investment satisfies

$$
e^{*} \cdot \exp e^{*}=\frac{25}{n} \cdot\left(v^{n}-R^{n}\right) .
$$

Set search engine's reserve price $R=0.25$ (currently, the advertisers' valuation range is $v \in[0.25,1]$ ). Substitute $R=0.25$ into (5.1), we draw $e^{*}(v, n)$ in Figure 1 which illustrates the optimal investment function with their valuation and the number of the advertisers when $R=0.25$.

From Figure 1, we can clearly see that when $v=0.25$, no matter how the number of advertisers changes, the optimal investment $e^{*}=0$, namely $e^{*}(v=R)=0$. Given search engine's reserve price, for advertisers with a certain valuation $(v \in(0.25,1])$, the greater the number of advertisers, the less their optimal investment, which verifies (ii) of Corollary 3.4. At the same time, as can be seen from Figure 1, the greater the valuation is, the more significant the reduction will be. 


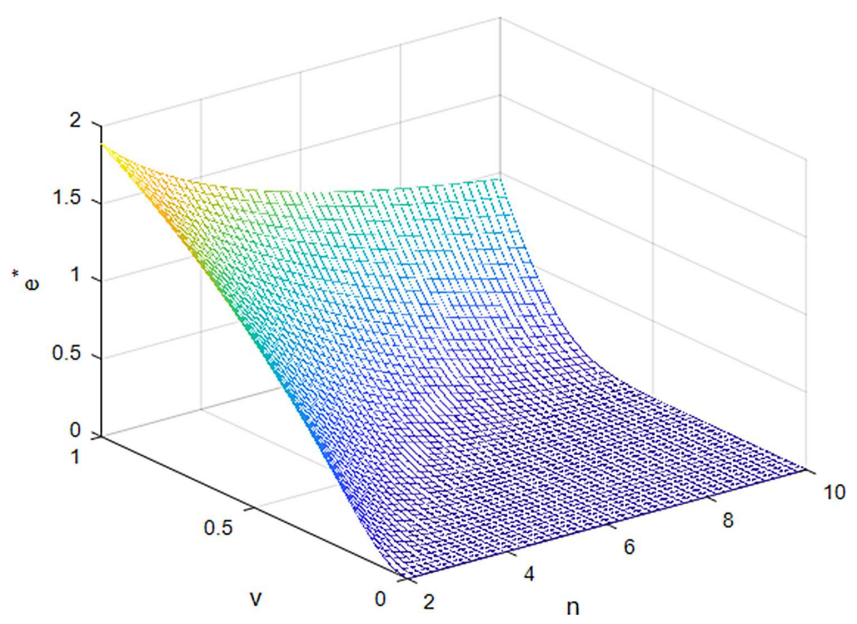

FIgURE 2. The optimal investment with $v$ and $n(R=0)$.

Then we set search engine's reserve price $R=0$ and get $e^{*}(v, n)$ when $R=0$ in Figure 2 .

Similarly, as (ii) of Corollary 3.4 expounds, advertisers' optimal investment is decreasing with the number of advertisers in Figure 2. Comparing Figure 1 with Figure 2, we can declare that when the advertiser's valuation is slightly greater than $0.25, e^{*}$ in Figure 1 (when $R=0.25$ ) is less than that in Figure 2 (when $R=0$ ). This intuitively indicates that the greater the search engine's reserve price is, the smaller $e^{*}$ is, which verifies (i) of Corollary 3.4. Furthermore, as the advertiser's valuation increases, the reduction caused by the increased reserve price will be smaller.

\subsection{The utility-maximizing reserve price and expected utility of search engine}

Under our parameter setting, the expected utility of search engine can be described as

$$
U_{\mathrm{SE}}=\int_{R}^{1}(2-\exp (-e)) \cdot v^{n-1} \cdot\left(\frac{n-1}{n} v+\frac{R^{n}}{n} v^{1-n}\right)^{1-r_{s}} \mathrm{~d} v .
$$

Then, on the basis of Proposition 4.1, we can derive the utility-maximizing reserve price from the equation set as follows:

$$
\begin{aligned}
& -y[e(v=R)] \cdot R^{n-r_{s}}+\int_{R}^{1}\left(-\frac{\exp (-2 e) \cdot R^{n-1}}{\frac{1}{25}+\exp (-e) \cdot \frac{v^{n}-R^{n}}{n}}\right) \cdot v^{n-1} \cdot\left(\frac{n-1}{n} v+\frac{R^{n}}{n} v^{1-n}\right)^{1-r_{s}} \mathrm{~d} v \\
& +\int_{R}^{1} y(e) \cdot\left(1-r_{s}\right) \cdot\left(\frac{n-1}{n} v+\frac{R^{n}}{n} v^{1-n}\right)^{-r_{s}} \cdot R^{n-1} \mathrm{~d} v=0, \quad \text { and } e^{*} \cdot \exp e^{*}=\frac{25}{n} \cdot\left(v^{n}-R^{n}\right) .
\end{aligned}
$$

Finally, the maximum of search engine's expected utility can be obtained by (5.2).

When the number of advertisers and the degree of search engine's risk aversion take different values (for a positive integer $n$ and $r_{s} \in[0,1)$ ), specific value of $R_{\mathrm{SE}}^{*}$ and the maximum of search engine's expected utility $U_{\mathrm{SE}}$ can be obtained, as shown in Table 1 (retain three decimal places).

Under our parameter setting, the following conclusion can be obtained from Table 1.

(1) Search engine's utility-maximizing reserve price decreases in the degree of search engine's risk aversion.

The higher the degree of search engine's risk aversion is, the more conservative the reserve price setting will be to ensure that the advertisement slot can be sold at a profitable bid. Therefore, a more risk-averse search engine 
TABLE 1. Comparison of $R_{\mathrm{SE}}^{*}$ and $U_{\mathrm{SE}}$ under Different $n$ and $r_{s}$.

\begin{tabular}{|c|c|c|c|c|c|c|c|c|}
\hline \multirow{2}{*}{$\begin{array}{l}n \\
r_{s} \\
\end{array}$} & \multicolumn{2}{|c|}{$n=2$} & \multicolumn{2}{|c|}{$n=3$} & \multicolumn{2}{|c|}{$n=4$} & \multicolumn{2}{|c|}{$n=5$} \\
\hline & $R_{\mathrm{SE}}^{*}$ & $U_{\mathrm{SE}}$ & $R_{\mathrm{SE}}^{*}$ & $U_{\mathrm{SE}}$ & $R_{\mathrm{SE}}^{*}$ & $U_{\mathrm{SE}}$ & $R_{\mathrm{SE}}^{*}$ & $U_{\mathrm{SE}}$ \\
\hline$r_{s}=0$ & 0.425 & 0.350 & 0.445 & 0.276 & 0.453 & 0.245 & 0.525 & 0.217 \\
\hline$r_{s}=0.25$ & 0.400 & 0.410 & 0.408 & 0.331 & 0.425 & 0.278 & 0.432 & 0.235 \\
\hline$r_{s}=0.5$ & 0.305 & 0.485 & 0.318 & 0.392 & 0.409 & 0.317 & 0.398 & 0.259 \\
\hline$r_{s}=0.75$ & 0.189 & 0.648 & 0.216 & 0.462 & 0.311 & 0.351 & 0.365 & 0.278 \\
\hline$r_{s}=0.9$ & 0.082 & 0.768 & 0.155 & 0.512 & 0.282 & 0.378 & 0.298 & 0.301 \\
\hline
\end{tabular}

tends to lower the reserve price to avoid auction risk. This is consistent with the conclusion of the relationship between auctioneer's risk aversion and reserve price in traditional auctions. Waehrer and Rothkopf [31] argued that in the first-price and second-price auction, sellers with higher degree of risk aversion will reduce their optimal reserve price setting. $\mathrm{Hu}$ et al. [16] also explored that under the first-price auction model, the greater the risk aversion of the seller, the lower the reserve price.

(2) The search engine's utility-maximizing reserve price decreases in the number of advertisers.

As we know, reserve price excludes advertisers whose bid is less than the reserve price. The larger the number of advertisers is, the more advertisers participate in the auction. Search engine will ensure that more advertisers participate in the bidding by lowering the reserve price. The fierce competition among advertisers is beneficial to search engine utility.

(3) Search engine's expected utility increases in the degree of search engine's risk aversion.

The degree of search engine's risk aversion has two opposing effects on search engine's expected utility: First, search engine with greater degree of risk aversion lower the utility-maximizing reserve price, which tends to reduce search engine's expected utility by decreasing the advertiser's bid. Second, the lower the reserve price, the more the advertiser's investment, as well as the corresponding user's click, which tends to enhance the search engine's expected utility. Under the parameter setting in our study, the resolution of such a tradeoff is that the increase of the search engine's utility caused by the increase of the advertiser investment will cover the loss brought by the advertisers' lowering the bid. Consequently, the expected utility of the search engine will increase.

(4) Search engine's expected utility decreases in the number of advertisers.

Similarly, the number of advertisers also has two opposing effects on search engine's expected utility. The greater the number of advertisers, the more intense the competitive pressure and the higher the advertiser's bid. On the contrary, advertisers' investment will decrease, as well as the corresponding clicks of advertisements. Under our parameter setting, the loss of search engine's utility caused by a decrease in clicks is greater than the increase due to the raise of advertiser bids. At this time, the expected utility will reduce. This is new conclusions drawn from our model which endogenous investment is introduced and portrayed in total clicks.

\subsection{The impact of reserve price on search engine's expected utility}

For a fixed number of advertisers $n=2$ and risk aversion parameter $r_{s}=0.25$, the search engine's expected utility is $U_{\mathrm{SE}}=\int_{R}^{1}(2-\exp (-e)) \cdot v \cdot\left(\frac{1}{2} v+\frac{R^{2}}{2} v^{-1}\right)^{0.75} \mathrm{~d} v$. How search engine's expected utility $U_{\mathrm{SE}}$ changes with reserve price is shown in Figure 3 below.

From Figure 3 we can see, when search engine sets no reserve price $(R=0)$, the search engine's expected utility is 0.38 . When $R$ increases approximately to 0.4 , the search engine's utility gradually increases (increasingly larger) to a maximum of 0.41 , where $R=0.4$ is the optimal reserve price for the search engine to maximize 


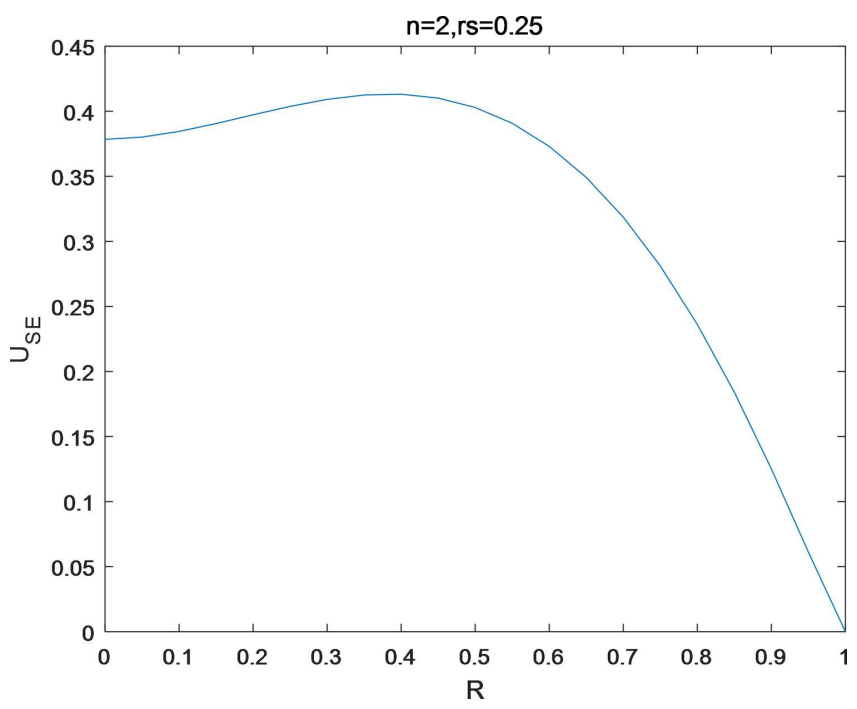

FIGURE 3. The effect of reserve price on search engine's expected utility.

expected utility. As $R$ continues to rise, search engine's expected utility begins to decline, and the rate of decline is faster and faster, until $R=1$, the utility is 0 (at this time no advertisers participate in the auction). Under different $n$ and $r_{s}$, we examine and discover that the general fluctuation trend about search engine's expected utility on the reserve price is consistent. In a word, setting the reserve price appropriately contributes to increase the expected utility, however, if the reserve price is set too high, search engine's expected utility will be lost. That is, there is an upper bound on search engine's utility when increasing the reserve price, so, search engine cannot always increase utility by increasing reserve price.

\subsection{Analysis of social welfare}

It is interesting to find that the efficient reserve price for maximizing the social welfare is zero regardless of value of $n$ and $r_{s}$, i.e. $R_{\mathrm{SW}}^{*}=0$ in our parameter setting. When the search engine does not set a reserve price, it can maximize the efficiency of the entire auction.

Under the parameter setting above, for a fixed number of advertisers $n=2$ and risk aversion parameter $r_{s}=0.25$, the social welfare is

$$
U_{\mathrm{SW}}=\int_{R}^{1}(2-\exp (-e)) \cdot v \cdot\left(\frac{1}{2} v+\frac{R^{2}}{2} v^{-1}\right)^{0.75} \mathrm{~d} v+\int_{R}^{1}(2-\exp (-e)) \cdot v \cdot\left(\frac{1}{2} v-\frac{R^{2}}{2} v^{-1}\right) \mathrm{d} v-\int_{R}^{1} \frac{e^{2}}{50} \mathrm{~d} v .
$$

Figure 4 below illustrates how social welfare changes with reserve price.

In Figure 4 we can intuitively draw that the efficient reserve price for maximizing social welfare is 0 . Contrast Figure 4 with Figure 3 , when $R=0.4$ (utility-maximizing reserve price), $U_{\mathrm{SE}}=0.41$ and $U_{\mathrm{SW}}=0.58$. When $R=0$ (efficient reserve price), $U_{\mathrm{SE}}=0.38$ and $U_{\mathrm{SW}}=0.65$. The loss of 0.03 in search engine's utility results in an increase of 0.07 in social welfare, which demonstrates that the increment of social welfare is much larger than the reduction of search engine's utility.

Efficient reserve price will allow more advertisers to participate in the bidding of investment, and enhance the performance level and social welfare of the entire advertising auction system. Despite the loss of some search engine's utility, the efficient auction mechanism will bring more social welfare. Therefore, the choice of social 


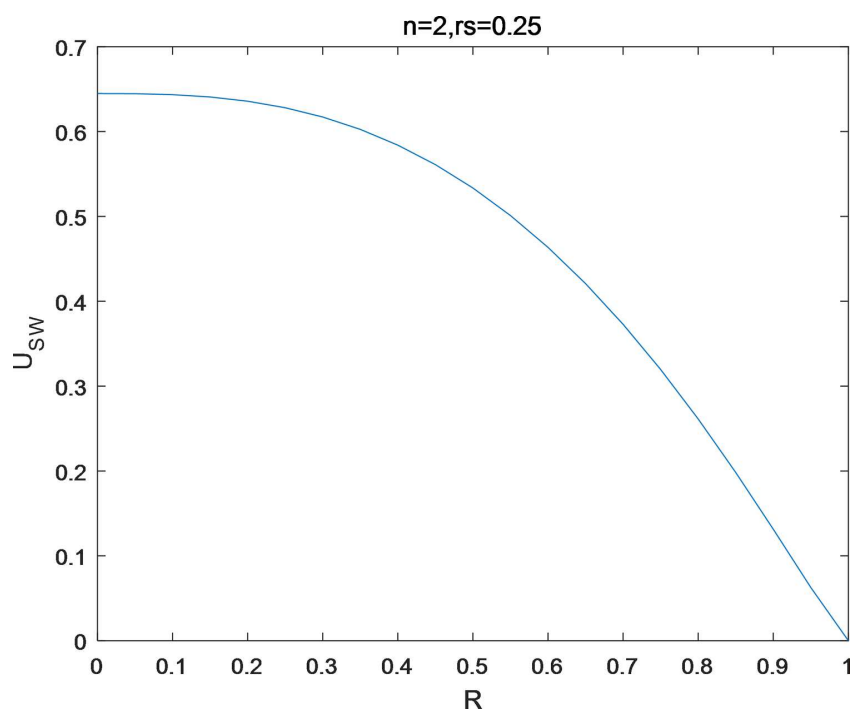

FIgURE 4. The effect of reserve price on social welfare.

welfare auction mechanism is more appropriate and profound, which is conducive to healthy and long-term development of the entire sponsored search auction market.

\section{CONCLUSiOn}

Our research extends the risk-neutral search engine to risk aversion and introduces advertisers' endogenous investment in keyword auction. We construct a first-price auction model and explore the equilibrium bidding strategy and the optimal investment decision-making of advertisers. Meanwhile, the paper deduces the determinant solution for search engine's reserve price which maximizes the expected payoff of search engine and social welfare separately. The study indicates the following results.

Firstly, regardless of the distribution which advertisers' private valuations follows, advertisers' equilibrium bidding function is monotonously increasing in bidders' valuations, the number of advertisers, as well as the reserve price.

Secondly, advertisers' optimal investment is negatively correlated with the reserve price and the number of advertisers, which is verified by both theoretical derivation and numerical analysis.

Thirdly, under our parameters setting, the numerical examples show that the utility-maximizing reserve price decreases with the search engine's risk aversion degree and the number of advertisers. Search engine's expected utility increases with the degree of risk aversion and decreases with the number of advertisers. Furthermore, we can declare that search engines can use reserve price as a regulatory tool to increase the utility. But there is an upper bound on search engine's utility.

Finally, using numerical examples, we find that the efficient reserve price is zero. When the search engine does not set reserve price, it can achieve maximum social welfare. For search engine, there exists a tradeoff between current utility and long-term development. Although setting no reserve price will lose a certain amount of search engine's utility, search engines can bring more social welfare and make the auction more efficient.

Our study expands the research on keyword auction theory in the fields of advertiser investment and riskaverse search engine. It provides a theoretical reference for advertisers to choose the optimal bid and investment, as well as for search engine to establish a balance between commercial interests and social welfare based on the substantial development. 
However, our study has some restrictions, and future work can be explored in depth. First, the coexistence of multiple keywords [11], multiple slots and multiple search engines can be taken into consideration. Second, considering that advertisers may be risk averse, introducing risk-averse advertisers will be an interesting research topic. Third, the degree of risk aversion is assumed as an exogenously given parameter in our study, so setting risk aversion parameter as random variable will be the direction of extending research.

Acknowledgements. The work was supported by Huazhong University of Science and Technology Special Funds for Development of Humanities and Social Sciences, the National Natural Science Foundation of China (Grant No. 71672071) and the Fundamental Research Funds for the Central Universities (Grant No. 2662017PY044).

\section{REFERENCES}

[1] A. Agarwal and T. Mukhopadhyay, The impact of competing ads on click performance in sponsored search. Inf. Syst. Res. 27 (2016) 538-557.

[2] G. Aggarwal, J. Feldman, S. Muthukrishnan and M. Pál, Sponsored search auctions with marconian users. Int. Workshop Internet Network Econ. $\mathbf{5 3 8 5}$ (2008) 621-628.

[3] L. Arozamena and E. Cantillon, Investment incentives in procurement auctions. Rev. Econ. Stud. 71 (2004) 1-18.

[4] A. Ayanso and A. Karimi, The moderating effects of keyword competition on the determinants of ad position in sponsored search advertising. Decis. Support Syst. 70 (2015) 42-59.

[5] F. Branco, Procurement favoritism and technology adoption. Eur. Econ. Rev. 46 (2002) 73-91.

[6] J.Q. Chen, D. Liu and A.B. Whinston, Auctioning keywords in online search. J. Marketing 73 (2009) $125-141$.

[7] J.Q. Chen, J. Feng and A.B. Whinston, Keyword auctions, unit-price contracts, and the role of commitment. Prod. Oper. Manage. 19 (2010) 305-321.

[8] D. Colucci, N. Doni and V. Valori, Information policies in procurement auctions with heterogeneous suppliers. J. Econ. 114 (2015) 211-238.

[9] J.C. Cox, V.L. Smith and J.M. Walker, Theory and individual behavior of first-price auctions. J. Risk Uncertainty 1 (1988) 61-99.

[10] Y.Y. Cui, S.L. Zou and Z.J. Ma, Auction-based resource allocation game under a hierarchical structure. Int. J. Syst. Sci. 47 (2016) 360-373.

[11] X. Du, M. Su, X. Zhang and X. Zheng, Bidding for multiple keywords in sponsored search advertising: keyword categories and match types. Inf. Syst. Res. 28 (2017) 711-722.

[12] B. Edelman and M. Schwarz, Optimal auction design and equilibrium selection in sponsored search auctions. Am. Econ. Rev. 100 (2010) 597-602.

[13] P. Esö, An optimal auction with correlated values and risk aversion. J. Econ. Theory 125 (2005) 78-89.

[14] H. Fang and X. Tang, Inference of bidders risk attitudes in ascending auctions with endogenous entry. J. Econ. 180 (2014) $198-216$.

[15] L. Friedman, A competitive-bidding strategy. Oper. Res. 4 (1956) 104-112.

[16] A. Hu, S.A. Matthews and L. Zou, Risk aversion and optimal reserve prices in first- and second-price auctions. J. Econ. Theory 145 (2010) 1188-1202.

[17] F. Kelly, P. Key and N. Walton, Efficient advert assignment. Oper. Res. 64 (2016) 822-837.

[18] C. Kocabasoglu, C. Prahinski and R.D. Klassen, Linking forward and reverse supply chain investments: the role of business uncertainty. J. Oper. Manage. 25 (2007) 1141-1160.

[19] D. Liu, J.Q. Chen and A.B. Whinston, Ex ante information and the design of keyword auctions. Inf. Syst. Res. 21 (2010) 133-153.

[20] J. Lu and I. Perrigne, Estimating risk aversion from ascending and sealed-bid auctions: the case of timber auction data. J. Appl. Econ. 23 (2008) 871-896.

[21] E. Maskin and J. Riley, Optimal auctions with risk averse buyers. Econometrica 52 (1984) 1473-1518.

[22] S. Matthews, Comparing auctions for risk averse buyers: a buyer's point of view. Econometrica 55 (1987) $633-646$.

[23] R.P. McAfee and J. McMillan, Auctions and bidding. J. Econ. Literature 25 (1987) 699-738.

[24] M. Ostrovsky and M. Schwarz, Reserve prices in internet advertising auctions: a field experiment. Res. Papers 2054 (2009) 59-60.

[25] J.W. Pratt, Risk aversion in the small and in the large. Econometrica 32 (1964) 122-136.

[26] J.G. Riley and W.F. Samuelson, Optimal auctions. Am. Econ. Rev. 71 (1981) 381-392.

[27] A.E. Schlosser, T.B. White and S.M. Lloyd, Converting web site visitors into buyers: how web site investment increases consumer trusting beliefs and online purchase intentions. J. Marketing $\mathbf{7 0}$ (2006) 133-148.

[28] R. Stephanie and W.S. Patrick, Reserve prices in auctions as reference points. Econ. J. 117 (2007) $637-653$.

[29] M. Sundararajan and I. Talgam-Cohen, Prediction and welfare in ad auctions. Theory Comput. Syst. 59 (2016) 664-682.

[30] W. Vickrey, Counterspeculation, auctions, and competitive sealed tenders. J. Finance 16 (1961) 8-37.

[31] K. Waehrer and M.H. Rothkopf, Auction form preferences of risk-averse bid takers. RAND J. Econ. 29 (1998) $179-192$.

[32] A. Watts, Two ways to auction off an uncertain good. J. Econ. 119 (2016) 1-15. 